\title{
Sobre la universalidad del Derecho internacional de los derechos humanos
}

\section{José Antonio Pastor Ridruejo}

Profesor emérito de Derecho internacional en la Universidad Complutense. Antiguo juez del Tribunal europeo de derechos humanos

SUMARIO: 1 . Planteamiento.- 2. Sobre La existencia De noRmas De DERECHO INTERNACIONAL GENERAL EN EL DERECHO INTERNACIONAL DE LOS DERECHOS HUMANOS. - 3. SOBRE EL CARÁCTER IMPERATIVO O DE IUS COGENS DEL DERECHO INTERNACIONAL DE Los Derechos humanos.- 4. Acerca del Carácter ERGA OMNES DEL DERECHO INTERNACIONAL DE LOS DERECHOS HUMANOS.- 5. UNA CONCESIÓN AL PARTICULARISMO: LA DOCTRINA DEL MARGEN DE APRECIACIÓN DEL TRIBUNAL EUROPEO DE DERECHOS HUMANOS. - 6 . CONCLUSIONES.

\section{Planteamiento}

1.1. De gran interés y sumamente discutida es la cuestión de si todos los habitantes del planeta deben gozar de los mismos derechos y libertades fundamentales o si, por el contrario, la lista y nivel de disfrute de esos derechos y libertades tienen que ver con la historia, religión, cultura, grado de desarrollo e ideología política reinantes en cada país o grupos de países. Se trata de la dialéctica entre la universalidad y el particularismo de los derechos humanos, planteada desde hace tiempo no solo en el plano académico sino también en el político. Porque si el debate doctrinal ha ocupado muchas páginas de monografías y artículos de revista, la dimensión política del problema ha 
aflorado con frecuencia en foros intergubernamentales. Ofrecemos un ejemplo tomado de la realidad. Y es que si en un foro de esa clase como la Asamblea General de las Naciones Unidas o la Comisión (Consejo desde el 14 de marzo de 2006) de derechos humanos de la organización mundial se censura la situación de los derechos humanos existente en un país determinado (digamos por seguir con un ejemplo real que se trata de un país de fundamentalismo islámico) no es inusual que sus representantes aleguen como defensa que se están aplicando parámetros normativos que no corresponden a la concepción de los derechos fundamentales imperante en ese país. Dicho de otro modo, esos representantes están sustentando una concepción particularista del Derecho internacional de los derechos humanos. Y que el debate tiene un importante componente político lo demuestran asimismo las posiciones adoptadas por las diversas delegaciones estatales en la Conferencia de las Naciones Unidas sobre los derechos humanos celebrada en Viena en 1993. Allí se adoptó una declaración final que leída en su integridad no deja de presentar ambigüedades, pues si de una parte se afirma que el carácter universal de los derechos y libertades no ofrece dudas, de otra parte se hace constar la importancia de las particularidades nacionales y regionales, así como de los diversos patrimonios históricos, culturales y religiosos ${ }^{1}$.

1.2. Ofrecemos en estas líneas una reflexión personal sobre el debate en cuestión. Y siendo indudablemente el Derecho internacional de los derechos humanos un capítulo más del Derecho internacional -y en modo alguno una pieza fragmentada o desgajada del mismo ${ }^{2}$ - , vamos a tomar en consideración y a poner en relación tres de sus

${ }^{1}$ La bibliografía sobre el debate es amplísima. Entre la española, puede verse especialmente con referencia a la Conferencia de Viena de 1993, C. VILLAN DURAN: "Significado y alcance de la universalidad de los derechos humanos en la Declaración de Viena". Revista española de Derecho internacional, 1994, vol. 2, pp. 505 ss.

2 Opinión que fundamento en "Droit international et Droit international des droits de l'homme, Unité ou fragmentation". Liber Amicorum Luzius Wildhaber, Basilea, 2007 
grandes conceptos básicos, a saber: $1^{\circ}$ ) el concerniente al modo de formación y ámbito de aplicación de las normas consuetudinarias generales de la disciplina, que son las que conforman el llamado Derecho internacional general; $2^{\circ}$ ) el relativo a las normas internacionales imperativas 0 de ius cogens y, $3^{\circ}$ ) el que trata de la significación precisa y de la relación con los anteriores conceptos de las llamadas obligaciones erga omnes.

\section{Sobre la existencia de noRmas de Derecho INTERNACIONAL GENERAL EN EL DERECHO INTERNACIONAL DE LOS DERECHOS HUMANOS}

2.1. Las fuentes ampliamente predominantes en el Derecho internacional de nuestros días, y muy particularmente en el Derecho internacional de los derechos humanos, son los tratados o convenciones. En el ámbito de este último sector de la disciplina, se trata de instrumentos que enumeran y definen los derechos fundamentales que los Estados partes se comprometen a respetar y hacer respetar. Añadamos que por lo común, esos mismos instrumentos establecen mecanismos de protección cuya naturaleza y eficacia son muy distintas según los casos. Digamos también que en ocasiones, aunque concertados entre Estados, los instrumentos en cuestión no asumen la naturaleza de tratado sino la de acuerdos de caballeros, declaración de intenciones o memoranda de entendimiento, lo que quiere decir que no despliegan efectos vinculantes desde el punto de vista jurídico ni se introducen en los Derechos internos. Su obligatoriedad solo tiene lugar en los planos moral y político. Sería el caso entre otros del Acta Final de Helsinki, adoptada el 1 de agosto de 1975 en el marco de la Conferencia sobre seguridad y cooperación en Europa.

En cualquier caso, el gran interrogante es si existen en materia de la salvaguarda de los derechos humanos normas consuetudinarias de carácter general. La respuesta a la pregunta reviste por supuesto interés científico y académico, pero además y sobre todo importancia en el terreno 
del Derecho positivo, puesto que de ser afirmativa estaríamos ante obligaciones exigibles jurídicamente a los Estados en el campo de los derechos fundamentales con independencia de cualquier vinculación de orden convencional.

2.2. A los efectos de la indagación propuesta, recordemos en primer lugar la definición de la norma consuetudinaria que hace el artículo 38 del Estatuto de la Corte internacional de justicia. Se habla en él de "la costumbre internacional como prueba de una práctica generalmente aceptada como Derecho". Parece necesario recordar asimismo algunas nociones básicas sobre la costumbre como fuente del Derecho internacional ${ }^{3}$. La primera es que, según los juristas romanos y como resulta del artículo mencionado del Estatuto de la Corte, de la jurisprudencia y de la práctica interestatal, la norma consuetudinaria consta de dos elementos: el material, o práctica constante y uniforme; y el espiritual u opinio iuris sive necessitatis, que es la convicción de la obligatoriedad de la práctica. En cuanto al ámbito de aplicación de la costumbre general, el punto de vista de la jurisprudencia internacional, práctica y doctrina, es que obliga a todos los Estados, hayan o no participado en su creación, con excepción de aquellos que durante el periodo formativo se hayan opuesto de manera expresa y continuada. Es decir, la costumbre general vincula a todos los Estados de la comunidad internacional, salvo a los llamados objetores persistentes. Digamos también que si en el Derecho internacional clásico, la consolidación de la práctica tenía lugar a lo largo de mucho tiempo, y que era esa consolidación la que acababa haciendo surgir el elemento espiritual o convicción de su obligatoriedad, en el Derecho internacional contemporáneo el proceso de formación de la costumbre es justamente el inverso. Desde la creación de las Naciones Unidas y la proliferación de conferencias mundiales de codificación del

${ }^{3}$ Entre la amplísima bibliografía, puede consultarse sobre la norma consuetudinaria y su evolución, Jose Antonio PAStor Ridruejo, Curso de Derecho internacional público y organización es internacionales, Madrid, Tecnos, 2006, pp. 69 ss. 
Derecho internacional, es frecuente que la opinio iuris se conforme en esos foros multilaterales y que impulse en lapsos de tiempo relativamente breves la aparición de la práctica y, en definitiva, la formación rápida de la norma consuetudinaria. Lo que quiere decir que desde hace ya unas cuantas décadas el centro de gravedad de la costumbre internacional está más en el elemento espiritual que en el material. Es también de destacar que la jurisprudencia internacional admite la existencia de costumbres negativas, es decir, de normas consuetudinarias que obliguen a una abstención, pero ello siempre que conste la convicción jurídica del deber de esa abstención. Esta última precisión es relevante en el tema que nos ocupa, puesto que tratándose de derechos civiles y políticos, el componente mayor y más significativo de las correspondientes obligaciones de los Estados es de respeto y abstención; por ejemplo, respetar el derecho a la vida no practicando ejecuciones sumarias, o no efectuar actos de tortura. Pero hay que aclarar inmediatamente que tratándose de esta primera generación de los derechos humanos - los civiles y políticos-, pesan también sobre los Estados obligaciones positivas: la de garantizar su disfrute. Es muy significativo a este respecto el tenor literal del artículo $2^{\circ}$ del Pacto de Naciones Unidas de 1966 sobre derechos civiles y políticos ${ }^{4}$. Por su parte el Tribunal europeo de derechos humanos ha construido una interesante jurisprudencia sobre las obligaciones positivas de los Estados

2.3. Con estos elementos de juicio, vamos a buscar la respuesta a la pregunta formulada líneas atrás, a saber, si existen normas consuetudinarias generales en materia de derechos humanos. Un primer dato favorable a esa existencia es un dictum de la Corte internacional de justicia

${ }^{4}$ Dice el párrafo $1^{\circ}$ del artículo en cuestión: "Cada uno de los Estados partes en el presente Pacto se compromete a respetar y garantizar a todos los individuos que se encuentren en su territorio y estén sujetos a su jurisdicción, los derechos reconocidos en el presente Pacto, sin distinción alguna de raza, color sexo, idioma, religión, opinión política o de otra índole, origen nacional o social, posición económica, nacimiento o cualquier otra condición social". Las cursivas son nuestras. 
en su opinión consultiva de 28 de mayo de 1951 acerca de las reservas a la Convención sobre genocidio. La Corte dijo, en efecto, que "los principios que fundamentan la Convención son principios reconocidos por las naciones civilizadas como obligatorios para los Estados incluso fuera de todo vínculo convencional"5. Trátese de principios generales del Derecho o de principios consuetudinarios generales del Derecho internacional —ambas posturas son a mi juicio defendibles - lo importante y cierto es que la Corte no limita al plano convencional la fuente normativa de obligaciones en materia de derechos humanos.

Luz clara en el tema es la que arroja la decisión emitida el 22 de octubre de 2002 por la Comisión interamericana de derechos humanos en el caso Michael Domíngues $c$. Estados Unidos de América ${ }^{6}$. Se trataba de determinar si personas menores (de 18 años) en el momento de la comisión del delito podían ser condenadas a muerte y ejecutadas. Como la Declaración al respecto de la Organización de Estados Americanos se limita a consagrar el derecho a la vida sin más especificaciones, la Comisión entendió que esa disposición debía ser interpretada con la ayuda del Derecho internacional consuetudinario. $\mathrm{Y}$ a los efectos de identificar en él a una norma determinada, declaró que era necesaria la concurrencia de cuatro requisitos, a saber: una práctica concordante por un ciertos número de Estados; un elemento temporal, es decir la repetición de la práctica por un periodo considerable de tiempo; la convicción de que esa práctica es la requerida por el Derecho internacional; y la aquiescencia general en la práctica de otros Estados.

Atendiendo a esos cuatro requisitos, la Comisión entendió que el Derecho internacional consuetudinario prohibía la condena a muerte y ejecución de personas que fuesen menores en el momento de la comisión del delito. Ahora bien, tras llegar a esta conclusión la Comisión hubo de ha-

${ }^{5}$ C.I.J., Recueil 1951, pp. 23-24.

${ }^{6}$ Inter-american Commission on Human Rigths, Michael Domingues v. United States of America, Case 12.285, Report No 62/02. 
cer frente al hecho de que los Estados Unidos de América eran un objetor persistente a en la materia. La Comisión conocía la jurisprudencia de la Corte internacional de justicia sobre el punto del objetor persistente, pero descartó esa doctrina al poner un énfasis especial en la idea de que la condena a muerte y ejecución de menores eran incompatibles con los estándares de decencia imperantes. Esto es, las medidas eran contrarias a una norma de Derecho internacional imperativo o de ius cogens.

Estoy de acuerdo con las líneas generales de la argumentación de la Comisión interamericana y con la conclusión de que el comportamiento que se imputa a los Estados Unidos de América no es conforme al Derecho internacional. Concuerdo también con la idea de que pueden existir normas consuetudinarias generales en materia de derechos humanos; y que la noción del ius cogens enerva la doctrina del objetor persistente, punto sobre el que volveré más adelante. Y señalo que a los efectos del debate de universalismo contra particularismo de los derechos humanos, las anteriores consideraciones llevan a afirmar de que en el Derecho internacional de los derechos humanos, al menos algunos de sus principios o normas obligan a todos los Estados sin que sea necesario para ello un vínculo convencional. Principios o normas que se inspiran consiguientemente en el principio de la universalidad.

2.4. Más clara está si cabe la existencia de principios o normas generales en un capítulo del Derecho internacional muy próximo al Derecho internacional de los derechos humanos. Estoy hablando del Derecho internacional humanitario; esto es, del sector de la disciplina que se ocupa de la protección de las víctimas de los conflictos armados $o$, si se quiere, de la salvaguarda de los derechos humanos en tiempo de guerra. En este campo abundan también los instrumentos convencionales, pero tanto estos como la más relevante jurisprudencia internacional admiten la existencia de principios o normas cuya obligatoriedad nada tiene que ver con compromisos convencionales. 
Así, las Convenciones de La Haya de 1907, las de Ginebra de 1949 y el Protocolo I de 1977 adicional a estas últimas convenciones de 1977 establecen para los casos de vacíos normativos o de denuncia de los instrumentos la llamada cláusula Martens. En el párrafo $2^{\circ}$ del artículo $1^{\circ}$ del instrumento últimamente citado, encontramos una formulación, redactada en término arcaicos pero sumamente claros, de la cláusula en cuestión. Es la siguiente:

En los casos no previsto en el presente Protocolo o en otros acuerdos internacionales, las personas civiles y los combatientes quedan bajo la protección y el imperio de los principios del derecho de gentes derivados de los usos establecidos, de los principios de humanidad y de los dictados de la conciencia pública.

En cuanto a la jurisprudencia, citemos un dictum de la sentencia de la Corte internacional de justicia recaída el 27 de junio de 1986 en el caso relativo a las actividades militares y paramilitares en Nicaragua y contra ella. Según este dictum existen "principios generales básicos de Derecho humanitario respecto de los que, a su juicio, las Convenciones de Ginebra constituyen en ciertos aspectos el desarrollo y en otros la expresión" 7 . La Corte se está refiriendo al artículo $3^{0}$ común a aquellas convenciones que es el que enuncia el catálogo de reglas mínimas aplicables a conflictos armados sin carácter internacional. Se trata de reglas - agrega la Corte - que corresponden a lo que ella denominó en 1949 "consideraciones elementales de humanidad" 8 . Finalmente en su opinión consultiva de 8 de julio de 1996 sobre la licitud de la amenaza o el empleo de las armas nucleares la Corte ha dicho que "estas reglas fundamentales se imponen de otro lado a todos los Estados, hayan o no ratificado los instrumentos convencionales que las expresan, puesto que constituyen principios inviolables del Derecho internacional consuetudinario" 9 .

7 C.I.J., Recueil 1986, p. 113.

8 C.I.J., Recueil 1949, p. 22.

${ }^{9}$ C.I.J., Recueil 1996, p. 28. 
3. Sobre El CARÁCTER IMPERATIVO O DE IUS COGENS DEL DeReCho INTERNACIONAL DE LOS DERECHOS HuManos

3.1. Las señas de identidad indiscutidas de las normas imperativas o de ius cogens son idénticas en todos los ordenamientos jurídicos y consisten en la no admisión de acuerdos en contrario y en la nulidad de los mismos en caso de celebración. Estamos por tanto ante normas no sometidas a la libre disposición de las partes, y de ahí que el ius cogens se contraponga al ius dispositivum.

Es este también el concepto del ius cogens internacional, que en formulación de Derecho positivo se recoge por vez primera en los artículos 53 y 64 de la Convención de Viena de 23 de mayo de 1969 sobre Derecho de tratados, instrumento en que es larga la lista de Estados partes (son 105, entre ellos España) aunque faltan de ella algunos miembros permanentes del Consejo de Seguridad de Naciones Unidas como Estados Unidos de América y Francia. Hay que señalar que en el artículo 53 citado se define, a efectos de la Convención, como norma imperativa de Derecho internacional a aquella "norma aceptada y reconocida por la comunidad internacional de Estados en su conjunto como norma que no admite acuerdo en contrario y que solo puede ser modificada por una norma ulterior de Derecho internacional general que tenga el mismo carácter". Lamentablemente, en la Conferencia de Viena que elaboró y adoptó la Convención, no fue posible llegar a un acuerdo sobre una definición sustantiva o material del ius cogens; es decir sobre los criterios que permitirían determinar de manera indiscutida cuándo se estaría en presencia de una norma de tal naturaleza. Pero de la definición convencional transcrita se deduce nítidamente que la identificación de una norma de ius cogens no requiere necesariamente su aceptación como tal por todos los Estados de la comunidad internacional; basta el reconocimiento por el conjunto de ellos. Se puede argumentar por consiguiente que en este campo no es operativa la idea del objetor persistente. Consiguientemente, una norma admitida como imperativa por el conjunto de la comunidad interna- 
cional conserva esta naturaleza incluso para los Estados que hayan podido objetar ese carácter. Idea esta que, como hemos visto en el apartado 2.2, inspiró a la Comisión interamericana de derechos humanos en su decisión de 22 de octubre de 2002 relativa al caso Michael Domíngues c. Estados Unidos de América.

3.2. De esta última decisión retengo ahora el dato del tema central de la misma, relativo nada menos que al más importante de los derechos fundamentales: el derecho a la vida. Esta circunstancia nos da una buena pista para ayudar a la determinación del carácter imperativo de una determinada norma, que es su inspiración indiscutida en valores éticos superiores o de primera magnitud. Y nadie discutirá que el derecho a la vida se inspira en esa clase de valores. Puede surgir entonces la pregunta de si todo el Derecho internacional de los derechos humanos esta integrado por normas de ius cogens A mi entender, la respuesta es negativa, y para fundamentarla voy a emplear un argumento a fortiori:

Los instrumentos convenciones generales sobre salvaguarda de los derechos humanos autorizan a los Estados partes a suspender o derogar temporalmente determinados derechos y libertades en situaciones excepcionales, como la guerra o algún peligro público que amenace la vida de la nación. Es el caso, por ejemplo del Pacto de Naciones Unidas sobre derechos civiles y políticos de19 de diciembre de 1966 (párrafo $1^{\circ}$ del artículo $4^{\circ}$ ); de la Convención europea de derechos humanos de 3 de noviembre de 1950 (artículo 15), y de la Convención interamericana de derecho humanos de 23 de noviembre de 1969 (artículo 27). Bien entendido, y esta precisión es de suma importancia, que esos instrumentos no autorizan la suspensión cuando se trata de derechos determinados. Es verdad que las Convenciones mencionadas discrepan en cuanto a la lista de derechos y libertades no susceptibles de suspensión o derogación temporal, pero todas ellas coinciden en prohibirlas para derechos tales como el derecho a la vida, y prohibición de la tortura, trabajos forzosos y esclavitud. 
Señalemos también que esos mismos instrumentos admiten en situaciones normales pero con sujeción a requisitos sumamente estrictos, que las autoridades estatales restrinjan en casos concretos el disfrute de determinadas libertades, como la de opinión, pensamiento, conciencia, religión, asociación, reunión y otras (Pacto de las Naciones Unidas, artículos 18, 19, 21 y 22; Convención europea, artículos 8, 9, 10 y 11; Convención interamericana, artícu$\operatorname{los} 12,165,16$ y 22 ).

Todo ello nos hace ver que la importancia de los derechos humanos es desigual y que conoce gradaciones. Hay en primer lugar derechos susceptibles de restricciones o interferencias en casos concretos incluso si se está en situaciones generales de normalidad; hay en segundo lugar derechos cuyo disfrute se puede suspender de modo general en situaciones excepcionales; y los hay finalmente que todos los instrumentos convencionales coinciden en considerar inderogables en cualquier tiempo y circunstancia. Constituyen estos últimos a mi juicio lo que a nivel universal se podría llamar el núcleo duro de los derechos fundamentales.

Haciendo ahora uso de la argumentación a fortiori que habíamos anunciado, cabe decir que si en situaciones normales o excepcionales los Estados pueden derogar o restringir unilateralmente el disfrute de determinados derechos, aún con sujeción a severas exigencias, podrían también en teoría concluir tratados que suspendan o limiten el goce de esos derechos, siempre, claro es, de manera excepcional y respetando las exigencias en cuestión. En teoría, insistimos, pues es poco verosímil la conclusión de esa especie de tratados, aunque no es del todo descartable que una disposición convencional produzca de modo indirecto y lateral un efecto contrario al ius cogens. Además, y como acabamos de comprobar, aquellas derogaciones temporales y restricciones sí son autorizadas en instrumentos convencionales generales. Todo ello demuestra que los principios o normas superiores que enuncian los derechos susceptibles de derogaciones o restricciones no tienen carácter imperativo. Sin embargo, esos mismos instru- 
mentos no autorizan en ningún caso la suspensión o derogación del núcleo duro de los derechos fundamentales; núcleo duro del que se puede predicar sin dificultad el carácter de ius cogens.

\section{ACerCa Del Carácter erga omNes DEL Derecho INTERNACIONAL DE LOS DERECHOS HUMANOS}

4.1. En un conoccido dictum de la sentencia de 5 de febrero de 1970 recaída en el caso de la Barcelona Traction (Bélgica c. España), la Corte internacional de justicia distingue dos clases de obligaciones internacionales, a saber, las que nacen en un Estado a favor de otro en el contexto de la protección diplomática, en primer lugar; y las que pesan sobre un Estado hacia la comunidad internacional en su conjunto, en segundo lugar. A esta últimas llama la Corte obligaciones erga omnes. Afirma además que tomando en consideración los intereses en juego todos los Estados tienen interés jurídico en la protección de esos derechos. Y agrega la Corte que "estas obligaciones se desprenden por ejemplo, en el Derecho internacional contemporáneo, de la puesta fuera de la ley de los actos de agresión y del genocidio, y también de los principios y las reglas concernientes a los derechos fundamentales de la persona humana, comprendiendo en ellos la protección contra la práctica de la esclavitud y la discriminación racial" ${ }^{10}$.

4.2. A la vista sobre todo del dictum de la Corte, un cierto sector doctrinal tiende a identificar las obligaciones erga omnes con las derivadas de las normas de ius cogens ${ }^{11}$. Y, ciertamente, existe un parentesco temático entre ambas nociones. Los ejemplos que pone la Corte en la sentencia Barcelona Traction confirman esta relación. Por su

10 C.I.J., Recueil 1970 , p. 31.

11 En España, muy particularmente por un reputado profesor de la pasada generación. Se trata de A. MiAJA DE LA MuELA, Aportación de la sentencia del Tribunal de La Haya en el caso Barcelona Traction (5 de febrero de 1970) a la jurisprudencia internacional, Cuadernos de la cátedra "J. B. Scott", Valladolid, 1970, pp. 73-74. 
parte, el proyecto de artículos de 2001 la Comisión de Derecho internacional subraya esa relación al establecer (artículo. 49) que por la violación grave de una norma imperativa de Derecho internacional todos los Estados son considerados como Estados lesionados y tienen por consiguiente calidad de reclamantes.

4.3. Entiendo con todo que el ámbito material de las obligaciones erga omnes es más amplio que el de las obligaciones derivadas de normas de ius cogens. O lo que es lo mismo, que existen obligaciones erga omnes que no derivan en rigor de obligaciones de ius cogens, afirmación fácil de comprobar en el Derecho internacional de los derechos humanos. En esta disciplina, y como se admite de modo indiscutido, las convenciones no se basan en la idea de la reciprocidad sino en el carácter absoluto u objetivo de las obligaciones establecidas ${ }^{12}$. No se trata únicamente de obligaciones asumidas por un Estado hacia los otros Estados partes, sino sobre todo y fundamentalmente de deberes hacia la comunidad internacional. Dicho de otro modo, de obligaciones erga omnes. Pero, como hemos dicho, entre esas obligaciones únicamente algunas tienen carácter de ius cogens. Son las que integran el núcleo duro de los derechos humanos, es decir, las que en ninguna circunstancia y de ninguna manera pueden ser objeto de suspensión o restricción. Pero las obligaciones susceptibles de suspensión o derogación temporal sí podrían ser objeto de acuerdos en contrario, siempre que respetasen, claro es, los requisitos exigidos para sus restricciones y suspensión. Al-

12 En el caso Irlanda c. el Reino Unido (sentencia de 18 de enero de 1978), el Tribunal Europeo de derechos humanos identificó con las siguientes palabras las características de los tratados sobre derechos humanos: "A diferencia de los tratados internacionales de tipo clásico, la Convención desborda el marco de la simple reciprocidad entre Estados contratantes. Más allá de una red de compromisos sinalagmáticos bilaterales, crea obligaciones objetivas que, según los términos del preámbulo, se benefician de una 'garantía colectiva'. En virtud de su artículo 24, permite a los Estados contratantes exigir el respeto de estas obligaciones sin tener que justificar un interés que derive, por ejemplo, de que una medida que denuncian ha lesionado a uno de sus nacionales..." C.E.D.H., Série $A, \mathrm{n}^{\circ} 25$. 
guien dirá con razón que este tipo de acuerdos es poco verosímil, aunque no es absolutamente descartable la hipótesis de que la cláusula de algún tratado conlleve la consecuencia de restringir de modo lateral o indirecto alguna libertad fundamental. A mi entender, tal restricción sería válida siempre que se sujetase a las exigencias del Derecho internacional de los derechos humanos tal como han sido codificadas en instrumentos convencionales. Es decir, que se trate de interferencias previstas en la ley y que en el marco de una sociedad democrática tiendan a conseguir las finalidades legítimas que las disposiciones pertinentes de esos mismos instrumentos especifican.

4.4. Señalemos por lo demás que la naturaleza erga omnes que nos ocupa tiene una manifestación muy clara en el terreno de los mecanismos internacionales de protección. Ciertamente, si la institución protectora se ha establecido en una convención y solo tiene competencia en los términos en ella establecidos - requiriendo por ejemplo que el Estado demandante y el Estado demandado sean parte en el instrumento- los terceros Estados no pueden reclamar por las violaciones. Pero si no existen limitaciones de este tipo, cualquier Estado se considera lesionado por las violaciones y se encuentra por consiguiente en condiciones de reclamar. $\mathrm{Y}$ esta consideración se manifiesta por ejemplo en foros intergubernamentales de las Naciones Unidas encargados de supervisar a nivel mundial el respeto de los derechos humanos. Así, en los procedimientos públicos especiales incoados por la Comisión de derechos humanos (Consejo de derechos humanos desde el 14 de marzo de 2006) todos los Estados integrantes del foro tienen voz y voto para el examen de situaciones de violaciones graves, masivas y sistemáticas de los derechos fundamentales existentes en algún país o respecto a una determinada categoría de derechos. Y lo mismo puede decirse de las actuaciones de la $3^{\text {a }}$ Comisión de la Asamblea General en los casos en que supervisa aquellas situaciones. Ello no es óbice, repetimos, de que cuando se trata de procedimientos creados por un instrumento convencional 
y activados por demandas, la capacidad para reclamar a un Estado solo se atribuya a Estados partes en ese instrumento. Es, entre otros, es el caso de las demandas interestatales previstas en el artículo 33 de la Convención europea de derechos humanos. Para que el Tribunal europeo de derechos humanos tenga competencia, Estado demandante y Estado demandado han de ser partes en la Convención.

\section{UNA CONCESIÓN AL PARTICULARISMO: \\ LA DOCTRINA DEL MARGEN DE APRECIACIÓN DEL TRIBUNAL EUROPEO DE DERECHOS HUMANOS}

5.1. Como es bien sabido, la responsabilidad primordial y principal de respetar y hacer respetar los derechos humanos y libertades fundamentales recae sobre las instancias políticas más próximas a los ciudadanos, es decir, sobre los Estados. El Derecho internacional de los derechos humanos tiene por tanto un carácter subsidiario porque sus normas e instituciones solo entran en juego cuando las instituciones nacionales fallan en el desempeño de su cometido principal ${ }^{13}$.

5.2. Añadamos a esta idea que la subsidiariedad del Derecho internacional de los derechos humanos tiene manifestaciones procesales y normativas. Ambas se aprecian muy bien en el funcionamiento de la institución internacional más eficaz para el respeto de los derechos fundamentales, que es sin duda el Tribunal europeo de derechos humanos. En el plano del procedimiento esas manifestaciones son dos: en primer término, la necesidad de agota-

${ }_{13}$ Sobre el principio de subsidiariedad del Derecho internacional de los derechos humanos, puede verse, entre la amplísima bibliografía, P. G. CARROZA, "Subsidiarity as a structural principle of International Human Rigts Law", en American Journal of International Law, 2003, vol. 1, pp. 38 ss; y J. A. PASTOR RIDRUEJO, "Le principe de subsidiarité dans la Convention europénne des droits de l'homme", en Internationale Gemeinschaft und Menchenrechte. Festchrift für Georg Ress zum 70. Geburstag am 21. Januar 2005, Carl Heymanns Verlag, 2005, pp. 1077 ss. 
miento de los recursos internos como condición de admisibilidad de las demandas; y en segundo término y en tanto que corolario de la primera manifestación, la obligación de los Estados de instaurar recursos eficaces al respecto en sus respectivos ordenamientos. Pero la manifestación de la subsidiariedad que ahora nos interesa es la normativa. Consiste en una importante construcción jurisprudencial que ha hecho aquél Tribunal. Es la doctrina del margen de apreciación. Efectivamente, cuando la formulación convencional de un derecho deja espacios de indefinición y no existe consenso o posición común entre los Estados partes en el instrumento sobre la solución adecuada a fin de llenar ese vacío, se entiende que son las instancias nacionales las más adecuadas y mejor colocadas para adoptar una solución al respecto. Damos algunos ejemplos jurisprudenciales para la mejor comprensión de la figura.

5.3. Así, en el caso Handy c. el Reino Unido (sentencia de 7 de diciembre de 1976) se trataba de la confiscación y multa al editor por la publicación de un libro considerado obsceno por las autoridades británicas. Pues bien, al examinar si se estaba ante una restricción a la libertad de expresión permitida por el párrafo 2 del artículo 10, el Tribunal, tras comprobar que entre los Estados partes en la Convención no existía una concepción europea común de la moral, declaró que la disposición citada concedía a las autoridades del Reino Unido un margen de apreciación para decidir lo que era contrario a la moral. La conclusión fue de no violación ${ }^{14}$. Ilustrativo también entre muchos otros es el caso Sahin c. Turquía, concerniente a la demanda de violación del artículo 11 de la Convención -libertad de religión - por la prohibición a las estudiantes portadoras del velo islámico de penetrar en la Universidad de Estambul. En su sentencia de 11 de noviembre de 2005 la Gran Sala del Tribunal dictó sentencia de no violación, apoyándose básicamente en la doctrina del margen de apreciación. El Tribunal tuvo en cuenta en su ratio deci-

${ }^{14}$ C.E.D.H., Série A, num. 24. 
dendi la autorización de restricciones o interferencias en la libertad de religión tal como las permite el párrafo $2^{\mathrm{o}}$ del referido artículo 11, dejando constancia de las posiciones divergentes en la materia de los Estados partes en la Convención y afirmando que la necesidad de preservar el principio del laicismo era esencial para la convivencia política en Turquía, de modo que entraba en el margen de apreciación de sus autoridades prohibir el porte del velo islámico femenino en el interior de los recintos universitarios. También para la aplicación del artículo 15 -suspensión de derechos en situaciones excepcionales- el Tribunal concede un amplio margen de apreciación de los Estados. Así lo hizo en la sentencia de 18 de enero de 1978 en el caso interestatal Irlanda c. el Reino Unido ${ }^{15}$. Por supuesto que podríamos citar muchísimas más sentencias que aplican el principio del margen de apreciación.

5.4. Me parece importante señalar ahora con un reputado tratadista en la materia y buen conocedor de la jurisprudencia del Tribunal ${ }^{16}$ que la doctrina del margen de apreciación no ha sido aplicada por el Tribunal en los casos relativos a violaciones de los artículos $2^{\circ}$ (derecho a la vida); $3^{\circ}$ (prohibición de la tortura y de tratos crueles, inhumanos y degradantes), y $4^{\circ}$ (prohibición de la esclavitud y de los trabajos forzados). Se trata justamente de derechos que de acuerdo al artículo 15 de la Convención europea no son nunca susceptibles de suspensión, ni siquiera en situaciones excepcionales. Derechos que nosotros hemos considerado como enunciados por normas de ius cogens y que tampoco son susceptibles de interferencias estatales en casos concretos. Y ese especial vínculo refuerza

15 C.E.D.H., Série A, num. 25.

16 J. CALLEWAERT, "Is there a Margin of Appreciation on articles 2, 3 and 4 of the Convention", Human Rigths Law Journal, 1998, vol. 19, num. 1 pp. 6 ss. Sobre la aplicación a otros artículos de la Convención, ver C. OveY, "The Margin of Appreciation and article 8 of the Convention". Ibid., pp. 10 ss.; S. C. Prebensen, "The Margin of Appreciation and articles 9, 10 and 11 of the Convention", Ibid., pp. 13 ss.; Y. WiNISDOERFFER, "Margin of Appreciation and Article 1 of Protocol No. 1", Ibid., 18 ss.; y J. SchoKkenBROECK, "The Prohibition of Discrimination in Article 14 of the Convention and the Margin of Appreciation”, Ibid., pp. 234 ss. 
la afirmación de que determinadas normas de la Convención tienen carácter imperativo, por no poder ser objeto de suspensión ni de la aplicación de la doctrina del margen de apreciación.

5.5. Por consiguiente, en la medida que la doctrina del margen de apreciación comporta la atribución a las autoridades de cada Estado de la facultad de determinar las consecuencias concretas y precisas de un derecho enunciado en términos genéricos, puede hablarse de una concesión al particularismo de los derechos humanos. Concesión no ilimitada, ciertamente, sino que como hemos visto está sometida al menos a tres requisitos: la existencia de un espacio de indefinición en la norma, la ausencia de posición común entre los Estados partes en el sistema, y la no aplicación a las normas que tengan carácter de ius cogens.

\section{Conclusiones}

Las conclusiones de estas apretadas reflexiones fluyen con facilidad de todo lo que llevamos dicho. Formuladas de modo sucinto son las siguientes.

La primera es que algunas de las normas del Derecho internacional de los derechos humanos forman parte de los principios generales del Derecho internacional consuetudinario, tienen además carácter de obligaciones erga omnes y en algunos casos la fuerza imperativa propia del ius cogens, condición esta última que a la hora de la formación de la costumbre enerva la operatividad de la figura del objetor persistente. Se trata de normas que se inspiran indiscutiblemente en el principio de universalidad.

La segunda conclusión es que en los supuestos en que la definición convencional de un derecho ofrece espacios de indefinición y sobre esos vacíos no existe posición común entre los Estados participantes en el sistema, la doctrina jurisprudencial del margen de apreciación constituye una concesión, clara aunque condicionada, al particularismo de los derechos humanos. 
En suma, y esta conclusión es una recapitulación de las anteriores, si algunos de estos derechos son de vigencia universal, el contenido de otros está condicionado por concepciones particulares que tienen que ver con las condiciones culturales, políticas, religiosas y otras imperantes en cada país o en cada región. Dicho de otro modo, universalismo y particularismo conviven en la disciplina del Derecho internacional de los derechos humanos. 